\title{
ChemComm
}

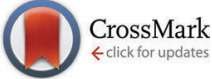

Cite this: Chem. Commun., 2015, 51, 9726

Received 7th April 2015

Accepted 12th May 2015

DOI: $10.1039 / \mathrm{c} 5 \mathrm{cc0} 02853 a$

www.rsc.org/chemcomm

\section{Lipase-modified $\mathrm{pH}$-responsive microgel-based optical device for triglyceride sensing $\dagger$}

\author{
Qiang Matthew Zhang, ${ }^{a}$ Darren Berg, ${ }^{b}$ Samuel M. Mugo* ${ }^{{ }^{b}}$ and Michael J. Serpe ${ }^{{ }^{a}}$
}

\begin{abstract}
Lipase-modified $\mathrm{pH}$-responsive poly( $\mathrm{N}$-isopropylacrylamide)-based microgels were synthesized. An optical device was subsequently fabricated by sandwiching the enzyme loaded responsive microgels between two thin Au layers, and their response to triolein, a model triglyceride, was investigated. The device's response depended on the triglyceride concentration, demonstrating its potential application as a triglyceride biosensor.
\end{abstract}

Triacylglycerols (TAGs) are triesters of glycerol and fatty acids, with typical chain lengths of 12-18 carbon atoms and degrees of unsaturation varying between 0 and $6 .{ }^{1}$ TAGs are a primary component of vegetable and animal lipids and hence a critical constituent of the human diet. TAGs are metabolized via hydrolysis by pancreatic lipase and biosynthesized into lipoproteins often classified as high-density lipoprotein (HDL) and low-density lipoprotein (LDL). The lipoproteins act as mobilizers of useful lipophilic entities such as cholesterol in the body. High levels of TAGs in serum, especially saturated TAGs, are directly linked to elevated LDL, which could create a highly atherogenic state ultimately leading to increased risk of heart attack. ${ }^{2,3}$ Also, from epidemiological studies, ${ }^{4}$ it is evident that elevated TAG levels in serum is associated with various cardiovascular diseases. For example, high concentrations of TAG can cause coronary diseases and disorders including diabetes mellitus nephrosis, liver obstruction and endocrine pathologies. ${ }^{5-7}$ Therefore, a sensitive and selective method to determine TAG concentration is essential for more effective and rapid clinical diagnosis.

Conventionally, TAGs are analysed by Lewis acid catalysed methylation followed by gas chromatography (GC) analysis; this is a lengthy analytical process. The IUPAC method for TAG analysis uses high performance liquid chromatography (HPLC) coupled with refractive index (RI) detection. ${ }^{8}$ Since this method

\footnotetext{
${ }^{a}$ Department of Chemistry, University of Alberta, Edmonton, AB, T6G 2 G2 Canada. E-mail: michael.serpe@ualberta.ca; Fax: +1 780492 8231; Tel: +1 7804925778

${ }^{b}$ Physical Sciences Department, MacEwan University, 10700-104 Avenue, Edmonton, AB, T5J 4S2 Canada. E-mail: mugos@macewan.ca; Tel: +1 7808877026

$\dagger$ Electronic supplementary information (ESI) available. See DOI: 10.1039/c5cc02853a
}

uses isocratic elution, analysis times are generally long, especially for mixtures containing long chain triglycerides. HPLC methods employing gradient elution with UV detection have provided faster separations than with refractive index. ${ }^{9}$ However, due to the low wavelengths necessary for triglyceride detection (for example, $210 \mathrm{~nm}$ ), UV analysis is very susceptible to baseline drift under gradient elution. In addition, the choice of mobile phase solvents is limited at lower wavelengths.

Recently, biosensors have attracted considerable attention as they can be made very sensitive, and specific, while many are simple and easy to use and can be very cost effective. ${ }^{10}$ Thus far, very few TAG biosensors that utilize immobilized TAG enzymes have been reported in the literature. ${ }^{11,12}$ Instead, existing biosensors typically take advantage of two main reactions. First, TAGs are hydrolyzed into glycerol and fatty acids in the presence of lipase. Then, the glycerol level is quantitatively determined via, e.g., colorimetry, fluorimetry and/or amperometry. ${ }^{13}$

The Serpe Group pioneered the development of a novel optical device (etalon), which can be fabricated by sandwiching a single layer of poly( $N$-isopropylacrylamide) (pNIPAm)-based hydrogel particles (microgels) between two semi-transparent thin gold $(\mathrm{Au})$ layers. These devices have found many applications, including for sensing and biosensing. ${ }^{14-19}$ The structure of an etalon can be seen in Scheme 1a. ${ }^{14,15}$ In short, these devices are constructed by "painting" a concentrated microgel solution onto a Au-coated glass substrate, followed by rinsing away the excess microgels not attached directly to the Au. After additional soaking and rinsing, the microgel layer was dried, and a subsequent layer of Au deposited on the microgel layer. These devices show visible color and unique multipeak reflectance spectra. The position and order of the peaks can be predicted from eqn (1):

$$
\lambda=2 n d \cos \theta / m
$$

where $n$ is the refractive index of the dielectric layer, $d$ is the mirror-mirror distance, $\theta$ is the angle of incident light relative to the normal, and $m$ (an integer), is the order of the reflected peak. Therefore, the size change of these microgels translates into a wavelength shift of the reflectance peaks. We point out 
(a)

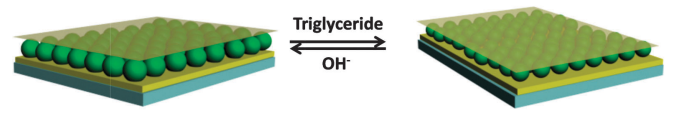

(b)

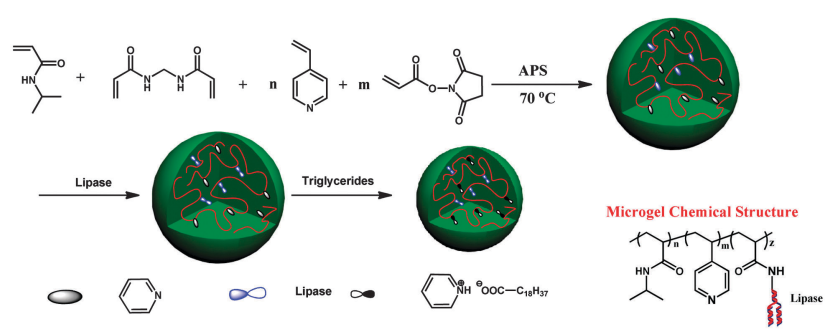

Scheme 1 (a) Etalon structure and responsivity, and (b) microgel synthesis scheme, and resulting microgel chemical composition.

here that the distance between the two Au layers dominates the etalons optical properties, and the refractive index change has a negligible influence on the peaks position.

In this submission, TAG-responsive etalons were fabricated; to accomplish this, lipase modified microgels were synthesized. Briefly, microgels with diameters of around $2 \mu \mathrm{m}$ (determined by dynamic light scattering (DLS)) were prepared by free radical precipitation polymerization of $N$-isopropylacrylamide (NIPAm), 4-vinylpyridine, $N, N^{\prime}$-methylenebis(acrylamide) (crosslinker) and $N$-acryloxysuccinimide, as shown in Scheme 1 . Lipase was covalently conjugated to microgels by reaction of the $-\mathrm{NH}_{2}$ on lipase with $\mathrm{N}$-oxysuccinimide on the microgels. Transmission electron microscope (TEM) images of these microgels are shown in Fig. 1. As can be seen, in both cases, the microgels are spherical, with average dry diameters of $1100 \mathrm{~nm}$ for enzyme free microgels (MG) and $900 \mathrm{~nm}$ for microgels loaded with lipase (MG-lipase) (via analysis of the microscope images). The microgel sizes were also characterized by DLS (Fig. S1 and S2, ESI $\dagger$ ). The DLS revealed hydrodynamic diameters of $2054 \pm 50 \mathrm{~nm}$ for MG and $1843 \pm 45 \mathrm{~nm}$ for MG-lipase in their swollen state (at $30{ }^{\circ} \mathrm{C}$ ). These values are averages of three repeat analyses of the microgels. The decrease in the microgel hydrodynamic diameter after lipase incorporation was attributed to the ability of lipase to crosslink inside the microgels. Specifically, lipase contains many amine groups $\left(-\mathrm{NH}_{2}\right)$ that can react with $\mathrm{N}$-oxysuccinimide of microgels. In this case, it can crosslink the microgels, causing them to decrease in diameter.

(a) $\mathrm{MG}$

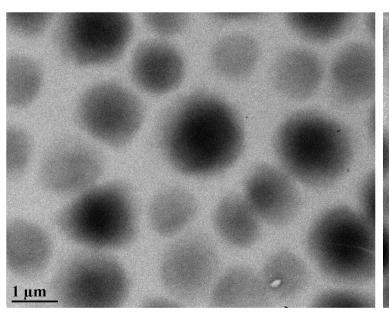

(b) MG-lipase

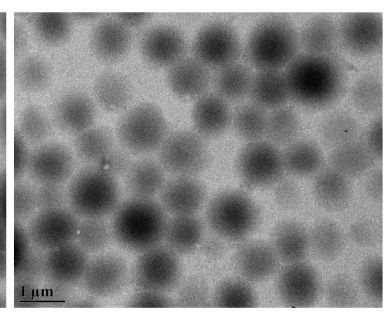

Fig. 1 TEM images of (a) lipase free microgels and (b) microgels with lipase loaded. Scale bar is $1 \mu \mathrm{m}$. (a)

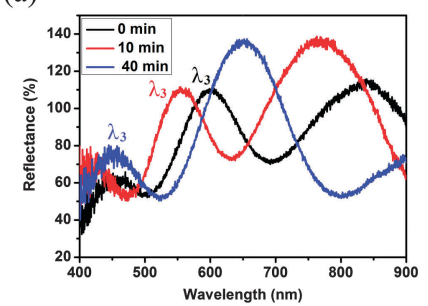

(b)

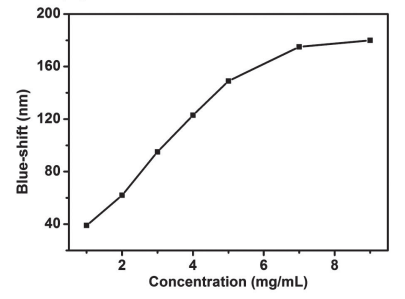

Fig. 2 (a) Reflectance spectra of etalon after exposure to triolein $\left(9 \mathrm{mg} \mathrm{mL}^{-1}\right)$ at $30{ }^{\circ} \mathrm{C}$ after the indicated times; (b) $\lambda_{3}$ blue-shift as function of triglyceride concentration.

The content of lipase in microgels was determined by Lowry protein assay $\left(\mathrm{ESI}^{\dagger}\right) .^{20}$ The result showed that lipase was quantitatively incorporated into microgels. Therefore, the content of lipase in microgels can be controlled by tuning $\mathrm{N}$-acryloxysuccinimide ratio in reactants.

Microgel-based etalons were subsequently constructed from the MG-lipase, and their responsivity to triolein (a model TAG) was investigated. The resulting reflectance spectra of the devices at $30{ }^{\circ} \mathrm{C}$ are shown in Fig. 2 . As can be seen, a characteristic multipeak reflectance spectrum was observed, with peaks at $453 \mathrm{~nm}, 600 \mathrm{~nm}$, and $850 \mathrm{~nm}$ at $30{ }^{\circ} \mathrm{C}$. From the relative positions of the reflectance peaks, and using eqn (1), the order $(m)$ of each peak can be calculated. The peak at $600 \mathrm{~nm}$ is $m=3$ (noted as $\lambda_{3}$ ), $\lambda_{4}$ is $453 \mathrm{~nm}$, while $\lambda_{2}$ is $850 \mathrm{~nm}$. When conducting these experiments, it is important to compare the same order peaks before and after introduction of TAG. As can be seen from Fig. 2a, $180 \mathrm{~nm}$ blue-shift of $\lambda_{3}$ occurred after exposure to triglycerides. We propose that triolein was hydrolyzed into long chain fatty acid $\left(\mathrm{C}_{18} \mathrm{H}_{37} \mathrm{COOH}\right)$ by lipase inside the microgels. The long chain fatty acid could subsequently attach to the microgels via acid-base reaction between fatty acid and the microgel's pyridine groups. The lipophilicity of long chain fatty acid increases the hydrophobicity of microgels, hence water was expelled from the microgels, and they collapsed. This resulted in a blue shift of the etalon's reflectance peaks.

As shown in Fig. 2b, as triolein concentration increases from $1 \mathrm{mg} \mathrm{mL}^{-1}$ to $7 \mathrm{mg} \mathrm{mL}^{-1}, \lambda_{3}$ blue-shifts from $39 \mathrm{~nm}$ to $175 \mathrm{~nm}$ and saturates at $\sim 7 \mathrm{mg} \mathrm{mL} \mathrm{m}^{-1}$. According to American Heart Association guidelines, the maximum safe level of TAG in the bloodstream is $1.5 \mathrm{mg} \mathrm{mL}^{-1} .^{21}$ Therefore, the device can respond in a physiologically relevant range. As a control, etalons were constructed from microgels without lipase, and exposed to triolein; these devices had no response to the addition (Fig. S3, ESI $\dagger$ ). This demonstrates that the blue-shift of the MG-lipase etalons was due to the hydrolysis of triglyceride by lipase. In another control, we exposed etalons constructed from pyridine-modified microgels to the fatty acid $\mathrm{C}_{18} \mathrm{H}_{37} \mathrm{COOH}\left(2 \mathrm{mg} \mathrm{mL}{ }^{-1}\right.$ final solution concentration). Upon addition, the etalon exhibited a $102 \mathrm{~nm}$ blue shift $\left(\lambda_{3}\right)$, Fig. S4 (ESI $\dagger$ ).

The response time of the devices was also investigated. This was done by exposing the devices to triolein, and monitoring the time it takes for the signal to stabilize. As can be seen in Fig. 3a, the response time decreased from $6 \mathrm{~h}$ to $40 \mathrm{~min}$, as the solution temperature increased from $22{ }^{\circ} \mathrm{C}$ to $30{ }^{\circ} \mathrm{C}$, and again 
(a)

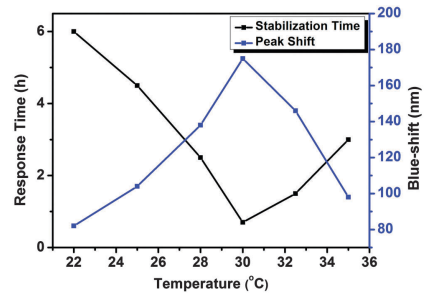

(b)

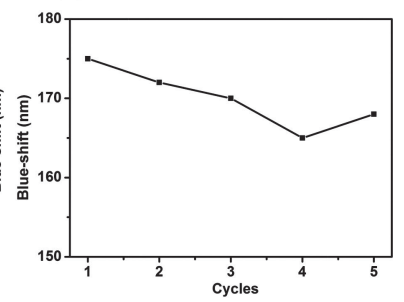

Fig. 3 (a) Response time and $\lambda_{3}$ blue-shift as function of temperature; (b) $\lambda_{3}$ blue-shift as function of the number of times used.

increased above $30{ }^{\circ} \mathrm{C}$. The improved response time as the solution temperature approaches $30{ }^{\circ} \mathrm{C}$ is hypothesized to be a result of two phenomena. First, it has been shown that the activity of lipase increases as temperature increases from 22 to $30{ }^{\circ}$ C. $^{22}$ Second, pNIPAm-based microgels are more able to collapse at temperatures approaching their lower critical solution temperature (LCST). Therefore, at temperatures approaching the LCST, the microgels will be very sensitive to changes in hydrophobicity. Furthermore, we hypothesize that the observed increase in the response time above the LCST is a result of the microgels becoming less porous in their collapsed state, making the lipase in the microgels less accessible, requiring more time for the enzymatic reaction and fatty acid generation. The fact that the microgels are already in a collapsed state above the LCST, also leads to the observed decrease in the magnitude of the response.

In another experiment, we determined if the device could be used multiple times to detect triolein. To "regenerate" the etalon after use, it was exposed to $\mathrm{K}_{2} \mathrm{HPO}_{4}-\mathrm{KH}_{2} \mathrm{PO}_{4}$ buffer solution ( $\mathrm{pH} 8$, $\left.\mathrm{H}_{2} \mathrm{O} / \mathrm{EtOH}=1: 1\right)$ to remove the long chain fatty acids $\left(\mathrm{C}_{18} \mathrm{H}_{37} \mathrm{COOH}\right)$ from the pyridine groups. As can be seen in Fig. $3 \mathrm{~b}$, the same device could be used at least 5 times without any significant loss of response $(\sim 3.4 \%)$.

In summary, pyridine-containing microgels were synthesized, and lipase covalently attached to the microgels. Etalons were fabricated by sandwiching these microgels between two thin $\mathrm{Au}$ layers, following established protocols. The resultant etalons were shown to exhibit a response to the presence, and concentration, of a triglyceride. We showed that the extent of the response varied systematically with triglyceride concentration, indicating its potential application as triglyceride biosensor. Due to their low cost $\sim 0.04 \mathrm{CAD}$ per square inch and ease of use, these devices could find real world applications in a clinical setting.

MJS acknowledges funding from the University of Alberta (the Department of Chemistry and the Faculty of Science), the Natural Sciences and Engineering Research Council of Canada (NSERC), the Canada Foundation for Innovation (CFI), the Alberta Advanced Education \& Technology Small Equipment Grants Program (AET/SEGP), Grand Challenges Canada and IC-IMPACTS. SMM acknowledges MacEwan Research for funding. MJS acknowledges Mark McDermott for the use of the thermal evaporator.

\section{Notes and references}

1 I. Polymer Labs, LCGC North Am., 2005, 54-55.

2 M. Castro Cabezas and D. W. Erkelens, Neth. J. Med., 2000, 56, 110-118.

3 P. Barter, Atheroscler. Suppl., 2005, 6, 15-20.

4 N. Sarwar, J. Danesh, G. Eiriksdottir, G. Sigurdsson, N. Wareham, S. Bingham, S. M. Boekholdt, K.-T. Khaw and V. Gudnason, Circulation, 2007, 115, 450-458.

5 R. K. Avramoglu, H. Basciano and K. Adeli, Clin. Chim. Acta, 2006, $368,1-19$.

6 P. R. Solanki, C. Dhand, A. Kaushik, A. A. Ansari, K. N. Sood and B. D. Malhotra, Sens. Actuators, B, 2009, 141, 551-556.

7 A. Vijayalakshmi, Y. Tarunashree, B. Baruwati, S. V. Manorama, B. L. Narayana, R. E. C. Johnson and N. M. Rao, Biosens. Bioelectron., 2008, 23, 1708-1714.

8 W. D. Pocklington and A. Hautfenne, Pure Appl. Chem., 1985, 57, 1515-1522.

9 J. P. Wolff, F. X. Mordret and A. Dieffenbacher, Pure Appl. Chem., 1991, 63, 1173-1182.

10 J. Kirsch, C. Siltanen, Q. Zhou, A. Revzin and A. Simonian, Chem. Soc. Rev., 2013, 42, 8733-8768.

11 J. Narang, N. Chauhan and C. S. Pundir, Int. J. Biol. Macromol., 2013, 60, 45-51.

12 A. Phongphut, C. Sriprachuabwong, A. Wisitsoraat, A. Tuantranont, S. Prichanont and P. Sritongkham, Sens. Actuators, B, 2013, 178, 501-507.

13 M. L. Moraes, L. Petri, V. Oliveira, C. A. Olivati, M. C. F. de Oliveira, F. V. Paulovich, O. N. Oliveira Jr and M. Ferreira, Sens. Actuators, B, 2012, 166, 231-238.

14 C. D. Sorrell and M. J. Serpe, Adv. Mater., 2011, 23, 4088-4092.

15 C. D. Sorrell, M. C. Carter and M. J. Serpe, Adv. Funct. Mater., 2011, 21, 425-433.

16 M. R. Islam and M. J. Serpe, Anal. Bioanal. Chem., 2014, 1-7.

17 M. R. Islam and M. J. Serpe, Anal. Chim. Acta, 2014, 843, 83-88.

18 L. Hu and M. J. Serpe, Chem. Commun., 2013, 49, 2649-2651.

19 Q. M. Zhang, W. Xu and M. J. Serpe, Angew. Chem., Int. Ed., 2014, 53, $4827-4831$.

20 S. M. Mugo and K. Ayton, J. Am. Oil Chem. Soc., 2013, 90, 65-72.

21 Triglycerides, Heart. Org., retrieved, 09-12-2012.

22 Y. Kuwahara, T. Yamanishi, T. Kamegawa, K. Mori, M. Che and H. Yamashita, Chem. Commun., 2012, 48, 2882-2884. 